\title{
Multiuser Water-filling in the Presence of Crosstalk
}

\author{
Wei Yu \\ Department of Electrical and Computer Engineering \\ University of Toronto \\ Toronto, Ontario M5S 3G4, Canada \\ Email: weiyu@comm.utoronto.ca
}

\begin{abstract}
Spectrum optimization is an important part of the design of interference-limited multiuser communication systems. While traditional water-filling provides a closed-form solution to the transmit optimization problem in a single-user system, the multiuser version of the problem often leads to nonconvex problem formulations which are difficult to solve. Motivated by high-speed parallel-link and digital subscriber line applications, this paper investigates two practical multiuser settings in which global or local optimal solutions to the multiuser spectrum optimization problems can be found efficiently. The first part of this paper considers a high-speed transmission system in which practical (but suboptimal) minimum-mean-squared-error linear equalizers (MMSE-LEs) are used at the receiver. The optimal single-user transmit spectrum in this case involves a modified water-filling solution. Surprisingly, such a modified water-filling spectrum can be shown to be near-optimal in a multiuser setting as well, if the direct-link and the crosstalk characteristics are symmetric and if crosstalk is reasonably small. Thus, for practical parallel-link systems using MMSE linear equalizers, the optimal single-user and multiuser spectra are nearly identical. The second part of this paper considers numerical techniques for solving a nonconvex multiuser rate maximization problem for digital subscriber line applications. A new ingredient in the proposed approach is a taxation scheme that takes into account the effect of interference between neighboring lines. This leads to a modified iterative water-filling algorithm which is capable of finding local optimum solutions to the multiuser spectrum optimization problem efficiently.
\end{abstract}

\section{INTRODUCTION}

Spectrum optimization is an important aspect of multiuser communication system design. While for traditional singleuser systems, spectrum optimization amounts to an optimal choice of bandwidth, power and modulation format for the maximization of single-user capacity, the design of multiuser systems also has to account for the effect of interference. Mathematically, while single-user spectrum optimization problem often leads to convex problem formulations which are analytically tractable, its multiuser counterpart often leads to nonconvex problems which are numerically difficult to solve. This paper makes progress on multiuser spectrum optimization problems by focusing on two specific scenarios in which efficient numerical algorithms exist. In the first part of the paper, we show that the formulation of transmit spectrum optimization problem depends on receiver design. While the use of an optimal receiver leads to a well-known water-filling solution, the use of a less-complex minimum-mean-squarederror (MMSE) linear receiver leads to a different water-filling spectrum. Surprisingly, this spectrum is near-optimal in some multiuser setting as well. In the second half of this paper, we focus on efficient numerical solutions to a nonconvex multiuser rate maximization problem in the presence of crosstalk interference. We show that a modified iterative water-filling algorithm can be used to arrive at local optimal solutions of the problem efficiently.

We are motivated to study the multiuser transmit spectrum optimization problem by high-speed parallel transmission line and digital subscriber line applications. (See Fig. 1.) In highspeed backplane communication systems, data transmissions occur on parallel links. The crosstalk between the links is a major transmission impairment. In these high-speed applications, the information-theoretical optimal multi-carrier modulation or decision-feedback equalization may be too complex to implement; suboptimal linear equalizers are often used. The first part of this paper tackles the optimal transmit power allocation problem in the presence of crosstalk when MMSE linear equalizers are used at the receiver.

The second part of the paper is motivated by applications in which information-theoretic achievable rate is the correct metric to optimize. This is the case for digital subscriber line systems where optimal receivers are used. The multiuser rate maximization problem for an interference channel is a wellknown nonconvex optimization problem. This paper provides numerical insights to the problem and proposes an efficient algorithm that aims to reach local optimal solutions of the problem.

\section{VARIATIONS OF WATER-FILLING}

Traditional water-filling refers to the shaping of the transmit spectrum for capacity maximization in a single-transmitter single-receiver Gaussian channel with additive colored noise. The water-filling solution is derived from a maximum mutual information argument; it is widely used in frequency-selective or intersymbol interference (ISI) channels, as well as in fading channels with adaptive modulation.

A key assumption in the derivation of water-filling spectrum is the use of an optimal receiver. However, when a suboptimal receiver such as an MMSE linear equalizer (MMSE-LE) is used, the water-filling spectrum needs to be suitably modified. In the following, we begin by reviewing the structures of optimal water-filling spectra for both optimal and suboptimal receivers. 


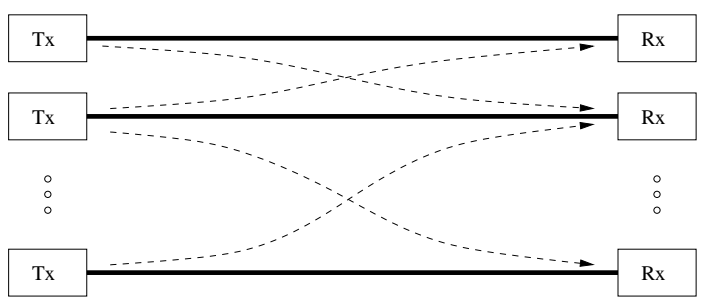

Fig. 1. A multiuser system in the presence of crosstalk. The optimal transmit spectrum depends on the receiver structure.

\section{A. Water-filling for Capacity Maximization}

The traditional justification for water-filling is based on mutual information maximization. In an ISI channel with a channel transfer function $H(f)$, the maximum achievable data rate subject to an input power constraint $P$ is the solution to the following optimization problem:

$$
\begin{aligned}
\operatorname{maxmize} & \int_{0}^{F} \log \left(1+\frac{|W(f)|^{2}|H(f)|^{2}}{\sigma^{2}}\right) d f \\
\text { subject to } & \int_{0}^{F}|W(f)|^{2} \leq P \\
& |W(f)|^{2} \geq 0,
\end{aligned}
$$

where the optimizing variable is input power spectral density, denoted as $|W(f)|^{2}, \sigma^{2}$ is the additive white Gaussian noise power level, and $F$ is the maximum frequency range used. This optimization problem is convex; it has the following simple solution:

$$
|W(f)|^{2}=\left(K-\frac{\sigma^{2}}{|H(f)|^{2}}\right)^{+},
$$

where $K$ is a constant chosen to satisfy the total power constraint. Equation (2) is referred to as the water-filling power allocation because $\frac{\sigma^{2}}{|H(f)|^{2}}$ can be thoughts of as the bottom of a bowl, $|W(f)|^{2}$ as the water filling the bowl, and $K$ as the water level.

In formulating the optimization problem (1) via mutual information, it is implicitly assumed that an optimal equalizer is used at the receiver ${ }^{1}$. This is true, for example, in an orthogonal frequency-division multiplex (OFDM) implementation where $H(f)$ is simply the channel gain and the optimal $W(f)$ is simply the power spectrum across the frequency tones. This can also true for single-carrier systems. As singlecarrier systems with decision-feedback equalizers can also be capacity achieving [1], the solution $W(f)$ obtained from (1) is the optimal transmit spectrum in this case as well. However, when the optimal transmit spectrum has a multi-band structure, multiple decision-feedback equalizers may have to be used.

\section{B. Water-filling with MMSE Linear Equalizer}

Clearly, water-filling is no longer the optimal transmit spectrum when suboptimal receivers are used. This is the case,

\footnotetext{
${ }^{1}$ In addition, (1) also assumes that use of capacity-achieving codes. However, the use of practical codes can be easily taken into account by including an SNR gap term in (1).
}
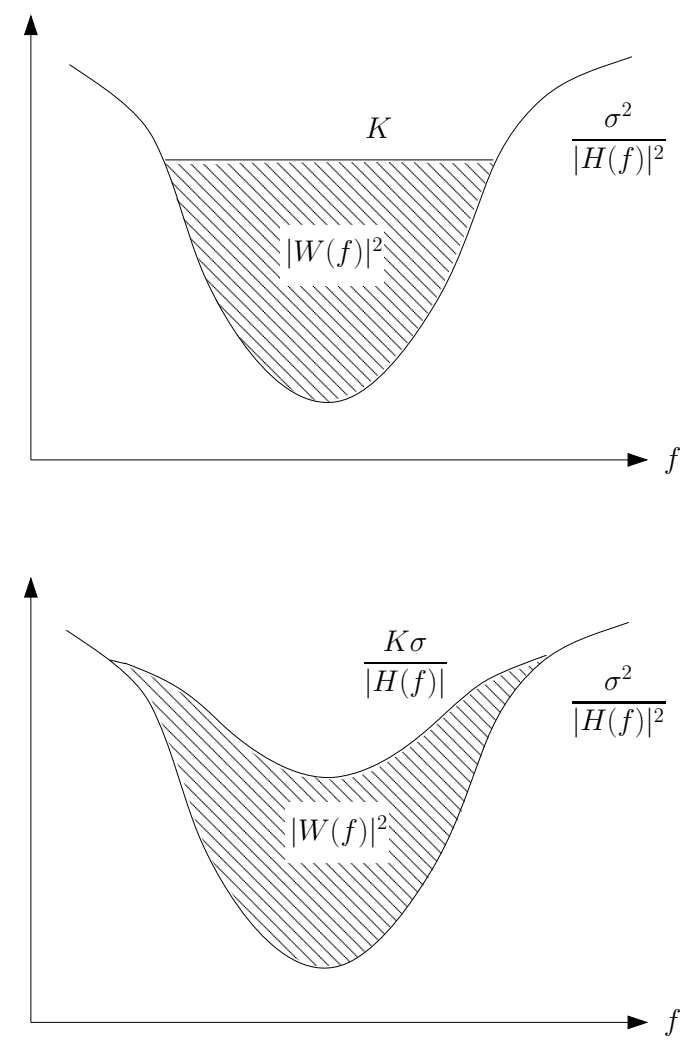

Fig. 2. Capacity-maximizing water-filling vs. MMSE-LE water-filling.

for example, when a linear equalizer is used. Consider the use of an MMSE-LE. Fixing the transmit spectrum $W(f)$, an MMSE-LE minimizes the total mean-squared error at the receiver. An interesting question is then: What is the optimal transmitter for such a suboptimal receiver? This problem has been formulated and solved in [2], where an analytic solution is shown to exist. We derive the solution below for the sake of completeness.

It is convenient to begin the derivation in matrix form. Consider an ISI channel with a Toeplitz matrix channel $H$ :

$$
\mathbf{y}=H \mathbf{x}+\mathbf{z}
$$

where $S_{z z}=\sigma^{2} I$. The transmit spectrum can be represented by a Toeplitz matrix $W$ in

$$
\mathbf{x}=W \mathbf{u} .
$$

Without loss of generality, we may set $S_{u u}=I$. The input power constraint then becomes $\operatorname{tr}\left(W W^{T}\right) \leq P$. The MMSE linear equalizer $L$ has the following form:

$$
\begin{aligned}
L & =S_{u y} S_{y y}^{-1} \\
& =W^{T} H^{T}\left(H W W^{T} H^{T}+\sigma^{2} I\right)^{-1}
\end{aligned}
$$

The minimum mean-squared error in this case is:

$$
\begin{aligned}
\text { MMSE } & =I-W^{T} H^{T}\left(H W W^{T} H^{T}+\sigma^{2} I\right)^{-1} H W \\
& =\left(I+W^{T} H^{T} \sigma^{-2} H W\right)^{-1}
\end{aligned}
$$


We now formulate the MMSE minimization problem subject to a power constraint. This is most easily done in the frequency domain. As both $H$ and $W$ are assumed to be Toeplitz, we now use their frequency-domain representations $H(f)$ and $W(f)$, respectively. In this case, the design problem becomes:

$$
\begin{aligned}
\text { minimize } & \int_{0}^{F} \frac{1}{1+\sigma^{-2}|W(f)|^{2}|H(f)|^{2}} d f \\
\text { subject to } & \int_{0}^{F}|W(f)|^{2} \leq P \\
& |W(f)|^{2} \geq 0,
\end{aligned}
$$

Interestingly, this problem also has an analytical solution. Following a Lagrangian approach, it is not difficult to show that the optimal transmit spectrum is [2]:

$$
|W(f)|^{2}=\left(\frac{K \sigma}{|H(f)|}-\frac{\sigma^{2}}{|H(f)|^{2}}\right)^{+}
$$

Comparing this solution with the capacity-maximizing waterfilling solution (2), it is interesting to note that the two are very similar, except that the water-filling level in (8) is no longer a constant. Instead, the MMSE-LE water-filling level is scaled by the inverse of the square root of the effective channel gain. Fig. 2 illustrates the difference between capacity-maximizing and MMSE-LE water-filling.

The capacity-maximizing water-filling solution always puts more power in high-SNR frequency bands in such a way that $|W(f)|^{2}+\frac{\sigma^{2}}{|H(f)|^{2}}$ is a constant. The MMSE-LE water-filling solution is less aggressive in spectrum shaping. The MMSELE water-filling process effectively depresses the water-filling level in the high-SNR frequency bands, and raises the waterfilling level in the low-SNR frequency bands. The MMSE-LE water-filling process is essentially a combination of channel inversion and water-filling.

\section{Multiuser WATER-FILLING With MMSE-LE}

The main objective of this paper is to derive the optimal multiuser water-filling solution in the presence of crosstalk. As mentioned earlier, in many high-speed multiline transmission systems, crosstalk interference between the lines is a dominant source of channel noise. Thus, the optimal transmit spectrum has to consider not only the maximization of each line's own rate (or the minimization of its own MSE), but also the effect of crosstalk interference each line emits to its neighboring lines. In this section, we consider the multiuser water-filling problem assuming MMSE-LE receivers.

In general, multiuser spectrum optimization often leads to nonconvex problem formulations, which are difficult to solve. In this section, we focus on a special, yet very practical, subclass of such problems for which an analytical solution is possible. This specific class includes parallel transmission line systems in which the direct channel transfer functions are identical for all lines and each line receives a same amount of crosstalk. This is the case, for example, for parallel backplane transmission line systems, where a set of equal-length lines are in close proximity to each other. The crosstalk channels are symmetric, and each line (except the first and the last line in the backplane) experiences the same amount of crosstalk. This is the scenario depicted in Fig. 1.

The optimal multiuser spectrum optimization problem in these scenarios simplifies significantly because of the symmetry. It is reasonable to assume that every transmitter and receiver must operate in the same way. In particular, the transmit power spectral density for each line must be identical. In addition, linear equalizers are assumed to be used at the receiver. We now ask: What is the optimal transmit spectrum with an MMSE-LE receiver?

By symmetric, the channel models for all users are identical and it simplifies to the following:

$$
\mathbf{y}=H \mathbf{x}+G \mathbf{v}+\mathbf{z}
$$

where $\mathbf{v}$ is the crosstalk signal and $G$ is the Toeplitz crosstalk channel. By assumption, $\mathbf{v}$ has the same power spectrum as $\mathrm{x}$. As before, $H$ is the Toeplitz matrix representing the direct channel. Let the transmit filter be:

$$
\mathbf{x}=W \mathbf{u}
$$

The total noise power spectrum then becomes $G W W^{T} G^{T}+$ $\sigma^{2} I$. Following the same derivation as in the single-user case, the minimum mean-squared error with an MMSE-LE is:

$$
\text { MMSE }=\left(I+W^{T} H^{T}\left(G W W^{T} G^{T}+\sigma^{2} I\right)^{-1} H W\right)^{-1} .
$$

Again, the minimization of the MMSE subject to power constraint is most easily done in the frequency domain. As $H$, $W$ and $G$ are all Toeplitz, the design problem then becomes:

$$
\begin{aligned}
\text { minimize } & \int_{0}^{F} \frac{1}{1+\frac{|W(f)|^{2}|H(f)|^{2}}{|G(f)|^{2}|W(f)|^{2}+\sigma^{2}}} d f \\
\text { subject to } & \int_{0}^{F}|W(f)|^{2} \leq P \\
& |W(f)|^{2} \geq 0,
\end{aligned}
$$

where $H(f), W(f)$ and $G(f)$ are the respective frequencydomain representations. The main result of this section is that the above problem has almost the same solution as in the single-user case.

Theorem 1: The optimal transmit spectrum that minimizes the mean-squared error in a symmetric crosstalk environment with MMSE linear equalizers at the receiver is

$$
|W(f)|^{2}=\frac{|H(f)|^{2}}{|H(f)|^{2}+|G(f)|^{2}}\left(\frac{K \sigma}{|H(f)|}-\frac{\sigma^{2}}{|H(f)|^{2}}\right)^{+}
$$

where $H(f)$ and $G(f)$ represent the direct and the total crosstalk channel gains, $\sigma^{2}$ is the additive background noise level, and $K$ is a constant chosen to satisfy the total power constraint.

Proof: The proof involves a manipulation of the KarushKuhn-Tucker condition for the optimization problem (12). Form the Lagrangian of (12) and take its derivation, we arrive at (14) at the top of the next page. After cancelation of terms, we get the optimality condition (13). 


$$
\frac{|G(f)|^{2}\left(\left(|G(f)|^{2}+|H(f)|^{2}\right)|W(f)|^{2}+\sigma^{2}\right)-\left(|G(f)|^{2}+|H(f)|^{2}\right)\left(|G(f)|^{2}|W(f)|^{2}+\sigma^{2}\right)}{\left(\left(|G(f)|^{+}|H(f)|^{2}\right)|W(f)|^{2}+\sigma^{2}\right)^{2}}=K
$$

The most remarkable aspect of this result is that the optimal transmit spectrum in a multiuser system with MMSE-LE is almost exactly the same as that of a single-user system with MMSE-LE, except by a scaling factor. The scaling factor is $\frac{|H(f)|^{2}}{|H(f)|^{2}+|G(f)|^{2}}$. In many practical systems of interests, the crosstalk channel gains are always much smaller than the direct channel gains. In these cases, the scaling factor is almost 1 and the optimal multiuser spectrum and the optimal singleuser spectrum are almost identical.

This is a surprising result, as the scaling factor is independent of the noise power level. One would expect that in situations where the crosstalk channel is much weaker than the direct channel, but where the background noise is even smaller, multiuser spectrum optimization would have produced a different transmit spectrum as compared to the single-user case. Yet, the two turn out to be near identical, even though the justifications for the single-user and the multiuser cases are very different.

\section{Multiuser Capacity Maximization}

The second part of this paper deals with a rate maximization problem for multiuser systems in the presence of crosstalk. Here, we assume that optimal receivers are used. In this case, the rate maximization problem becomes the maximization of mutual information in an interference environment.

Although the capacity region of the interference channel is still an open problem, in practical systems where the crosstalk channels are typically weaker than the direct channel, treating interference as noise is often the most practical communication strategy. This is the case for digital subscriber line (DSL) applications, which is the motivating example in this part of the paper.

When the crosstalk interference is regarded as noise, a $K$ user spectrum optimization problem for rate maximization becomes:

$$
\begin{array}{ll}
\max & \sum_{k=1}^{K} \alpha_{k} \sum_{n=1}^{N} \log \left(1+\frac{P_{k}(n)\left|H_{k k}(n)\right|^{2}}{\sigma^{2}+\sum_{j \neq k} P_{j}(n)\left|H_{j k}(n)\right|^{2}}\right) \\
\text { s.t. } & \sum_{n=1}^{N} P_{k}(n) \leq \mathbf{P}_{\mathbf{k}} \quad k=1, \cdots, K \\
& P_{k}(n) \geq 0 \quad k=1, \cdots, K
\end{array}
$$

Here, the objective function is a weighted rate maximization problem with weighting factor $\alpha_{k}$. We discretize the entire frequency range into $N$ tones, corresponding to that of a practical OFDM modulation system, where $H_{j k}(n)$ denotes the channel transfer function from user $j$ to user $k$ in tone $n$, and $P_{k}(n)$ denotes the power spectrum for user $k$ in tone $n$. The total power constraint for user $k$ is denoted as $\mathbf{P}_{\mathbf{k}}$. In a DSL application, the crosstalk channels cannot be assumed to be symmetric (i.e. $H_{i j}(n) \neq H_{j i}(n)$ in general.)
The multiuser rate maximization problem (15) has been considered extensively in the literature. An early idea, called iterative water-filling [3], calls for the use a greedy algorithm to arrive at an approximate solution to this problem. In the iterative water-filling algorithm, each user iteratively performs a single-user water-filling, while regarding interference as noise. As an update of each user's power spectrum changes the interference pattern for all other users, the water-filling process needs to be performed iteratively until the power spectra of all users converge. While efficient and very easy to implement, the iterative water-filling algorithm does not attempt to solve the weighted rate maximization problem optimally. Each user maximizes its own rate only, with no regard to other users' rates.

Fundamentally, the weighted rate maximization problem is a difficult problem to solve numerically, because of the nonconvex nature of the optimization objective. The best numerical algorithm to date is known as the optimal spectrum balancing (OSB) algorithm [4], which relies on a dualization of the power constraint using a Lagrangian multiplier and an exhaustive search over each user's power spectrum for each fixed value of Lagrangian multipliers. The dual optimization approach works despite nonconvexity, because the spectrum optimization problem is separable in the frequency domain and it satisfies an unusual property that its duality gap is zero in the limit of large $N$ [5]. Nevertheless, because the optimal spectrum balancing algorithm involves an exhaustive search over the user power, the complexity of OSB is exponential in $K$, which makes it impractical for systems with a large number of users.

The OSB algorithm depends crucially on the separability of the rate expression over frequency. Separability in this context means that frequency tones are independent of each other, and the power of each user in tone $n$ affects the aggregate rate only in tone $n$. While separability holds for (15), the rate expression of (15) is only an idealization. In practical OFDM systems where the received OFDM symbol from the direct channel and that from the crosstalk channel do not perfectly align with each other, intercarrier interference (ICI) results [6]. In this case, the total crosstalk interference for user $k$ in tone $n$ has the following expression [6]:

$$
\text { Crosstalk }=\sum_{j \neq k}\left(\sum_{m=-\frac{N}{2}}^{\frac{N}{2}-1} \gamma(m) P_{j}(n-m)\left|H_{j k}(n-m)\right|^{2}\right)
$$

where $\gamma(0)=1$ and $\gamma(m)=2 /\left(N^{2} \sin ^{2}(\pi m / N)\right)$, for $m \neq$ 1 , is the tone-to-tone coupling coefficient ${ }^{2}$ [6]. In this case,

\footnotetext{
${ }^{2}$ Note that due to the circulant structure of OFDM modulation, the tone indices in $H_{i j}(n)$ and $P_{j}(n)$ in (16) should be regarded as circulant, e.g. $P_{j}(-1)=P_{j}(N-1)$.
} 
the power of a user in tone $n$ affects its neighbors not only in tone $n$, but also in tones $n \pm 1, n \pm 2$, etc. The problem becomes a nonconvex optimization problem with a coupled utility function. The dual decomposition approach of the OSB algorithm no longer applies.

The objective of this part of the paper is to find an simple and effective algorithm to solve the multiuser spectrum optimization problem numerically. Our main idea is an iterative way of solving the KKT system of the nonconvex optimization problem, which can be interpreted as a modified iterative water-filling scheme capable of finding local optimal solutions to the problem efficiently. The proposed algorithm is useful in the presence of intercarrier interference as well.

Consider the Lagrangian of the optimization problem (15), dualized with respect to the power constraint:

$$
\begin{aligned}
\max & \sum_{k=1}^{K} \alpha_{k} \sum_{n=1}^{N} \log \left(1+\frac{P_{k}(n)\left|H_{k k}(n)\right|^{2}}{\sigma^{2}+\sum_{j \neq k} P_{j}(n)\left|H_{j k}(n)\right|^{2}}\right) \\
& +\sum_{k=1}^{K} \lambda_{k}\left(\mathbf{P}_{\mathbf{k}}-\sum_{n=1}^{N} P_{k}(n)\right) \\
\text { s.t. } & P_{k}(n) \geq 0, \quad k=1, \cdots, K .
\end{aligned}
$$

We can write down the KKT system of the optimization problem by taking the derivative of the above with respect to $P_{k}(n)$ :

$$
\frac{\alpha_{k}}{P_{k}(n)+\sum_{j \neq k} P_{j}(n) \frac{\left|H_{j k}(n)\right|^{2}}{\left|H_{k k}(n)\right|^{2}}+\frac{\sigma^{2}}{\left|H_{k k}(n)\right|^{2}}}=\lambda_{k}+t_{k}(n)
$$

where $t_{k}(n)$ is defined as follows:

$$
\begin{array}{r}
t_{k}(n)=\sum_{j \neq k} \frac{\alpha_{j} P_{j}(n)\left|H_{j j}(n)\right|^{2}}{P_{j}(n)\left|H_{j j}(n)\right|^{2}+\sum_{l \neq j} P_{l}(n)\left|H_{l j}(n)\right|^{2}+\sigma^{2}} . \\
\frac{\left|H_{k j}(n)\right|^{2}}{\sum_{l \neq j} P_{l}(n)\left|H_{l j}(n)\right|^{2}+\sigma^{2}}
\end{array}
$$

Solving the optimization problem (15) can now be thought of as solving the KKT system (18)-(19) along with the power constraints and positivity constraints on $P_{k}(n)$ and the usual complementary slackness conditions ${ }^{3}$.

The inclusion of the term $t_{k}(n)$ is the only difference between the usual iterative water-filling procedure and this new set of KKT systems. The term $t_{k}(n)$ summarizes the effect of interference that user $k$ causes to other users. This motivates us to propose a modified iterative water-filling algorithm for solving the KKT system.

Our main idea is to fix both $t_{k}(n)$ and the interference term from other users, denoted by $I_{k}(n)$, where

$$
I_{k}(n)=\sum_{j \neq k} P_{j}(n)\left|H_{j k}(n)\right|^{2}
$$

\footnotetext{
${ }^{3}$ When $P_{k}(n)=0$, the equality in (18) needs to be replaced by less than or equal to.
}

and solve for $\lambda_{k}$ and $P_{k}(n)$ using (18). We then update the $t_{k}(n)$ and $I_{k}(n)$ terms according to the newly obtained $P_{k}(n)$ and repeat the process until convergence.

This strategy is numerically efficient because (18) is essentially a modified water-filling step, which can be rewritten as:

$$
P_{k}(n)+\frac{I_{k}(n)}{\left|H_{k k}(n)\right|^{2}}+\frac{\sigma^{2}}{\left|H_{k k}(n)\right|^{2}}=\frac{\alpha_{k}}{\lambda_{k}+t_{k}(n)}
$$

Note that the water-filling level is modified by $\alpha_{k}$ and $t_{k}(n)$ on a per-tone basis.

Fixing $t_{k}(n)$ and $I_{k}(n)$, we propose the following modified water-filling procedure to find the optimal $P_{k}(n)$. The first step is to recognize that given $\lambda_{k}, P_{k}(n)$ can be obtained from (21) as follows:

$$
P_{k}(n)=\left(\frac{\alpha_{k}}{\lambda_{k}+t_{k}(n)}-\frac{I_{k}(n)+\sigma^{2}}{\left|H_{k k}(n)\right|^{2}}\right)^{+}
$$

Here, $\lambda_{k}$ is essentially the inverse of the water-filling level but modified by $t_{k}(n)$. Next, to find $\lambda_{k}$, we can sum (22) over $n$ and get

$$
\mathbf{P}_{\mathbf{k}}=\sum_{n}\left(\frac{\alpha_{k}}{\lambda_{k}+t_{k}(n)}-\frac{I_{k}(n)+\sigma^{2}}{\left|H_{k k}(n)\right|^{2}}\right)^{+}
$$

With $t_{k}(n)$ and $I_{k}(n)$ fixed, this is now an equation of a single variable $\lambda_{k}$. Further, the right-hand side of (23) is a monotonic function of $\lambda_{k}$. Thus, (23) can be solved efficiently via a onedimensional search (e.g. using bisection). After $\lambda_{k}$ is found, $P_{k}(n)$ can then be obtained from (22).

Note that when the optimal $P_{k}(n)$ involves using less power than the total power constraint $\mathbf{P}_{\mathbf{k}}$, the optimal $\lambda_{k}$ should be set to zero. This is the case when no positive $\lambda_{k}$ satisfies (23).

Once the optimum $\left(P_{k}(n), \lambda_{k}\right)$ is obtained for a given user $k$, we may then iterate over the users, taking into account the updated interference term $I_{k}(n)$, until the process converges. Finally, in an outer loop, we may then update $t_{k}(n)$ according to (19) and repeat the process until the entire KKT system is solved. The algorithm is summarized in the following:

\section{Modified Iterative Water-filling Algorithm}

1) Initialize $P_{k}(n)$ and $t_{k}(n)$.

2) Loop:

a) Fixing $t_{k}(n)$, loop over user $k$ :

i) Calculate $I_{k}(n)$ according to (20).

ii) Obtain $\lambda_{k}$ via a bisection on (23).

iii) Calculate $P_{k}(n)$ using (22).

Until $P_{k}(n)$ converges.

b) Update $t_{k}(n)$ using (19).

Until $t_{k}(n)$ converges.

The idea of using a term $t_{k}(n)$ to take into account the effect of interference each user causes onto others has been used in the past (e.g. [7] [8]). The main novelty of the proposed algorithm is the incorporation of the $t_{k}(n)$ term in the waterfilling process, which allows us to find the water-filling level $\lambda_{k}$ efficiently using bisection. 


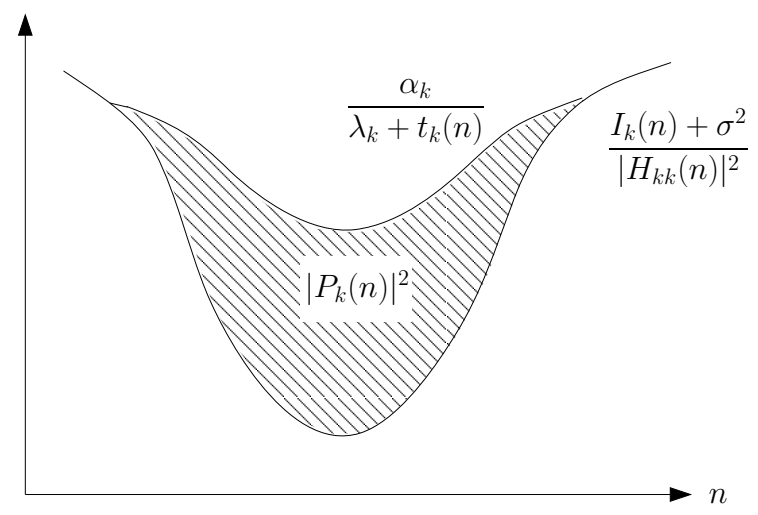

Fig. 3. Water-filling with a modified water level.

The $t_{k}(n)$ term can be interpreted as a taxation term. It lowers the water-filling level (and hence the transmit power spectrum density) in frequencies where the effect of its interference to other users is strong. This is done on a tone-by-tone basis. The outer loop sets the value of the taxation term. Each user responds by maximizing the weighted sum rate over its own power allocation $P_{k}(n)$. The weighting factor $\alpha_{k}$ also has an effect of adjusting the overall water-filling level.

As compared to previous work, the proposed algorithm has the following main advantages:

- In previous work, the update of $\lambda_{k}$ is always done via a subgradient method [4] [7] [8], which can be slow. The proposed bisection method is much more efficient.

- The proposed algorithm has an interpretation of being a modified water-filling process for each user as shown in Fig. 3. This facilitates distributed implementation, as $t_{k}(n)$ is the only information that needs to be exchanged among the users, while the interference term $I_{k}(n)$ can be measured by each modem in real time. In addition, a modification of the water-filling level is the only change that is required in implementation. This facilitates the implementation of discrete bit-loading.

- The modified iterative water-filling algorithm can be easily adapted to asynchronous DSL systems with intercarrier interference [6]. This is a key advantage as compared to OSB [4] or its derivatives [5] [9], and other previously proposed algorithm such as SCALE [10]. To account for intercarrier interference, the crosstalk term (20) only needs to be modified as (16). The taxation term (19) only needs to be modified as shown in (25) at the top of the next page ${ }^{4}$, while all other parts of the algorithm remain the same.

As typical in iterative-water-filling-like algorithms, the convergence proof is difficult to establish in full generality. It is possible to adopt an approach of [7] to show that under a weak-interference condition, the iterative modified waterfilling inner loop for a fixed $t_{k}(n)$ would converge. However,

${ }^{4}$ In (25), $\operatorname{SINR}_{j}(r)$ is defined to be the signal-to-interference-and-noise ratio for $j$ th user at the $r$ th tone.

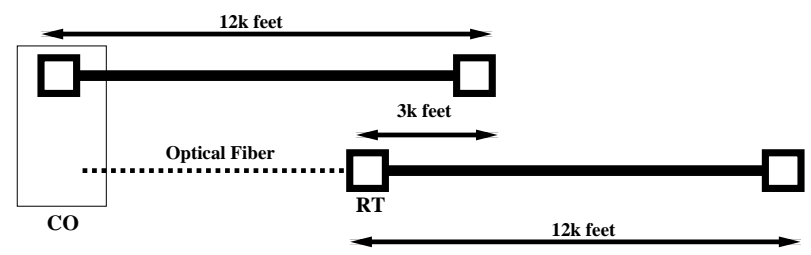

Fig. 4. Loop topology for two downstream ADSL users. User one is deployed from the central office (CO). User two is deployed from a remote terminal (RT). The RT user emits strong crosstalk to the $\mathrm{CO}$ user.

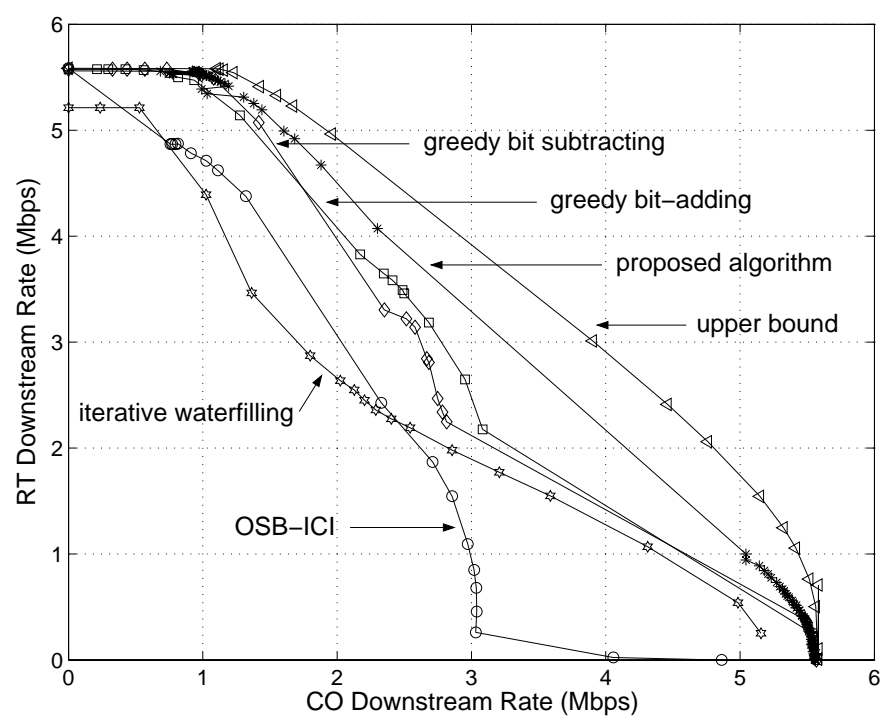

Fig. 5. Comparison between rate regions of the proposed algorithm and iterative water-filling [3], OSB [4], and two greedy algorithms proposed in [6] for the two-downstream-ADSL scenario with intercarrier interference. The OSB performs poorly because the optimal spectrum is derived assuming no ICI.

in practice, these weak-interference conditions are not always met, yet convergence is still observed.

The convergence of $t_{k}(n)$ in the outer loop is also not easy to establish. However, one may ensure convergence by slowing down the update of $t_{k}(n)$ by incorporating a memory term. For example, let $\hat{t}_{k}(n)$ be the taxation term as computed by (19), the following update rule can be used to slow down the update

$$
t_{k}^{\text {new }}(n)=\beta t_{k}^{\text {old }}(n)+(1-\beta) \hat{t}_{k}(n)
$$

for some $0<\beta<1$.

Finally, we note that since the proposed algorithm solves the KKT system directly. When it converges, it always converges to a KKT point, which is guaranteed to be at least a local optimum solution to the original problem (15).

Note that the proposed algorithm reduces to the original iterative water-filling algorithm [3] when the taxation terms are set to be $t_{k}(n)=0$. Thus, the proposed algorithm is attractive from an implementation point of view, as it allows legacy systems to be upgraded gradually. 


$$
\begin{aligned}
t_{k}(n)= & \sum_{j \neq k} \alpha_{j}\left|H_{k j}(n)\right|^{2} \sum_{r=-\frac{N}{2}}^{\frac{N}{2}-1} \frac{P_{j}(r)\left|H_{j j}(r)\right|^{2}}{P_{j}(r)\left|H_{j j}(r)\right|^{2}+\sum_{l \neq j} \sum_{m=-\frac{N}{2}}^{\frac{N}{2}-1} \gamma(m) P_{l}(r-m)\left|H_{l j}(r-m)\right|^{2}+\sigma^{2}} \\
& \frac{\gamma(r-n)}{\sum_{l \neq j} \sum_{m=-\frac{N}{2}}^{\frac{N}{2}-1} \gamma(m) P_{l}(r-m)\left|H_{l j}(r-m)\right|^{2}+\sigma^{2}} \\
= & \sum_{j \neq k} \alpha_{j}\left|H_{k j}(n)\right|^{2} \sum_{r=-\frac{N}{2}}^{\frac{N}{2}-1} \frac{\left[\operatorname{SINR}_{j}(r)\right]^{2}}{1+\operatorname{SINR}_{j}(r)} \cdot \frac{\gamma(r-n)}{P_{j}(r)\left|H_{j j}(r)\right|^{2}}
\end{aligned}
$$

\section{NUMERICAL Simulation}

In this section, we present a simulation example to illustrate the effectiveness of the proposed modified iterative waterfilling algorithm. We choose a particularly strong crosstalk scenario in asymmetric digital subscriber lines (ADSL) as shown in Fig. 4, where the line deployed from a remote terminal (RT) emits a strong crosstalk signal to the line deployed from a central office (CO). In addition, intercarrier interference is assumed to be present.

The achievable rate region for the proposed algorithm as compared to the iterative water-filling algorithm [3], the OSB algorithm [4], and two greedy algorithms proposed in [6] is presented in Fig. 5. As shown, because of the presence of ICI, the OSB algorithm performs rather poorly. This is expected as OSB optimizes spectrum assuming no ICI, and the optimized spectrum typically involves frequency-division multiplexing, which leads to poor performance when ICI is present. The iterative water-filling algorithm also does not perform particularly well, because it does not take into account the effect of strong interference from the RT user to the $\mathrm{CO}$ user. The two greedy algorithms proposed in [6] are based on local greedy subtraction or addition of bits starting from some initial spectrum. While outperforming OSB and iterative water-filling, they still do not perform particularly well. The proposed algorithm produces a larger achievable rate than all previous algorithms. In Fig. 5, an outer bound, which is computed without the ICI terms, is also plotted. Clearly, the outer bound is not achievable. Although the proposed algorithm only achieves local optimal solutions, the local optimum obtained appears to be fairly close to the global optimum for this particular example. Finally, we note that simulation experience shows that the proposed algorithm is also more efficient than OSB and the greedy algorithms proposed in [6].

\section{CONCLUding REMARKS}

While the performance of a single-user system is not necessarily a very sensitive function of the input spectrum, the performances of multiuser systems often are because of the presence of interference. For this reason, spectrum optimization has become an important part of the overall communications system design in many practical multiuser systems.
However, spectrum optimization in the presence of crosstalk interference is a challenging problem. For a general multiuser channel setting, the problem formulation leads to nonconvex structures which are often analytical intractable and numerically difficult to solve. This paper investigated two specific examples of practical importance in which either analytic or efficient numerical solutions are available. Interestingly, both solutions resembles a single-user water-filling solution, except with a modified water-filling level. These single-user-waterfilling-like solutions are attractive, because they are efficient, robust, and are often amendable to distributed implementation.

\section{ACKNOWLEDGMENT}

This work was supported in part by the Natural Science and Engineering Research Council of Canada, and in part by Huawei Technologies, China. The author wishes to thank Mr. Jun Yuan for performing the simulation in the paper.

\section{REFERENCES}

[1] J. M. Cioffi, G. P. Dudevoir, M. V. Eyuboglu, and G. D. Forney, "MMSE decision feedback equalizers and coding: Part I and II," IEEE Trans. Commun., vol. 43, no. 10, pp. 2582-2604, Oct. 1995.

[2] D. P. Palomar, J. M. Cioffi, and M. A. Lagunas, "Joint Tx-Rx beamforming design for multicarrier mimo channels: A unified framework for convex optimization," IEEE Trans. Signal Processing, vol. 51, no. 9, pp. 2381-2401, Nov. 2003.

[3] W. Yu, G. Ginis, and J. M. Cioffi, "Distributed multiuser power control for digital subscriber lines," IEEE J. Select. Areas Commun., vol. 20, no. 5, pp. 1105-1115, June 2002.

[4] R. Cendrillon, W. Yu, M. Moonen, J. Verlinden, and T. Bostoen, "Optimal multiuser spectrum balancing for digital subscriber lines," IEEE Trans. Commun., vol. 54, no. 5, pp. 922-933, May 2006.

[5] W. Yu and R. Lui, "Dual methods for nonconvex spectrum optimization of multicarrier systems," IEEE Trans. Commun., vol. 54, no. 6, pp. 1310-1322, June 2006.

[6] V. M. K. Chan and W. Yu, "Multiuser spectrum optimization for discrete multitone systems with asynchronous crosstalk," IEEE Trans. Signal Processing, 2006, submitted.

[7] J. Huang, R. Cendrillon, M. Chiang, and M. Moonen, "Autonomous spectrum balancing (ASB) for frequency selective interference channels," in IEEE Inter. Symp. Inf. Theory (ISIT), Seattle, U.S.A., 2006.

[8] J. Yuan and W. Yu, "Distributed cross-layer optimization of wireless sensor networks: A game theoretic approach," in Global Telecommunications Conf. (GLOBECOM), San Francisco, U.S.A., 2006.

[9] R. Cendrillon and M. Moonen, "Iterative spectrum balancing for digital subscriber lines," in IEEE Inter. Conf. Commun. (ICC), Seoul, Korea, 2005.

[10] J. Papandriopoulos and J. S. Evans, "Low-complexity distributed algorithms for spectrum balancing in multi-user DSL netwoks," in IEEE Inter. Conf. Commun. (ICC), Istanbul, Turkey, 2006. 